\title{
Optimized constitutive distributions visualized by lamina formulas
}

\section{Pedersen, Pauli; Pedersen, Niels Leergaard}

\section{Published in:}

Mechanics of Advanced Materials and Structures

Link to article, DOI:

10.1080/15376494.2016.1191097

Publication date:

2017

Document Version

Peer reviewed version

Link back to DTU Orbit

\section{Citation (APA):}

Pedersen, P., \& Pedersen, N. L. (2017). Optimized constitutive distributions visualized by lamina formulas. Mechanics of Advanced Materials and Structures, 24(5), 385-391.

https://doi.org/10.1080/15376494.2016.1191097

\section{General rights}

Copyright and moral rights for the publications made accessible in the public portal are retained by the authors and/or other copyright owners and it is a condition of accessing publications that users recognise and abide by the legal requirements associated with these rights.

- Users may download and print one copy of any publication from the public portal for the purpose of private study or research.

- You may not further distribute the material or use it for any profit-making activity or commercial gain

- You may freely distribute the URL identifying the publication in the public portal

If you believe that this document breaches copyright please contact us providing details, and we will remove access to the work immediately and investigate your claim 


\title{
Optimized constitutive distributions visualized by lamina formulas
}

\author{
Pauli Pedersen and Niels L. Pedersen \\ Department of Mechanical Engineering, Solid Mechanics \\ Technical University of Denmark \\ Nils Koppels Allé, Building 404, DK-2800 Kgs. Lyngby, Denmark \\ email: pauli@mek.dtu.dk and nlp@mek.dtu.dk
}

This paper is dedicated to Professor Carlos Mota Soares on his 70th birthday.

\begin{abstract}
For optimal design most parameters may be classified in size, shape and topology, such as simple density variables and parameters for surface description. Density and surface can be rather directly visualized. Extending the design to material design in sense of design of distributions of constitutive matrices, a practical visualization is more complicated but may be based on classical laminate analysis. In rotational transformation of constitutive matrices, some practical quantities are often termed invariants, but the invariance relates to an unchanged reference direction. Rotating this reference direction, the practical quantities do change and this point is clarified with derived rotational transformation for these practical quantities.
\end{abstract}

The theoretical and numerical background for design of optimal anisotropic constitutive matrices are presented. Then design results are applied in a $2 \mathrm{D}$ visualization of optimized constitutive matrices that are distributed in a finite element (FE) model where each element has a specific reference direction. The visualized distributions of physical quantities are stiffest material direction, material stiffest longitudinal constitutive component, level of anisotropy, absolute or relative shear stiffness and test of orthotropy. 
Keywords: constitutive anisotropy, optimality criteria, multiple load cases, sensitivity, strength, eigenfrequency, laminate formula.

\section{Introduction}

In free material optimization (FMO), the components of the constitutive matrices are optimized and they change in the space of a finite element (FE) model, i.e., they are distributed. The constraints for the non-dimensional description of these matrices are symmetry, positive definite and normalized to unit trace. The optimized constitutive matrices should be visualized, but this is not an easy task and different techniques are applied in the literature. From the authors point-of-view the visualization should be related to the most important physical quantities, and for $2 \mathrm{D}$ problems the traditional lamina analysis is found valuable.

In recent research simple formulas for design of constitutive matrices are obtained, related to different static as well as to eigenfrequency optimal design problems. It is shown that for quite different design objectives, the elastic energy density plays a major role and the results are expressed directly by the current strains, with unit matrix norms and separated from the local amount of material. In optimal design the name optimality criterion is used for a mathematical necessary condition for optimality. Two such criteria are involved in the present paper. Firstly a criterion for the components of non-dimensional constitutive matrices, describing the local anisotropy. This criterion gives directly the components, based on a current strain field. A second criterion for the density variables, i.e., for the material distribution among the elements. This criterion is the basis for a numerical redesign procedure that fulfills the criterion where size limits are not active.

Visualization of fields of $3 \times 3$, symmetric, positive definite constitutive matrices of 
unit norms are based on formulations from laminate theory. From laminate analysis, the formulation for rotational transformation of constitutive matrices is applied and is found useful. Practical parameters which usual are stated as invariants are an important part of this formulation but the notion invariants needs to be discussed, because the parameters depend on a specific reference direction. The visualized distributions of physical quantities are stiffest material direction, material stiffest longitudinal constitutive component, level of anisotropy, absolute or relative shear stiffness and orthotropy test.

Analysis and optimization may be performed without rotational transformations in a common coordinate system with the $x$-direction as reference (for 2D). However, the visualizations of the optimized results involve rotational transformation of material behavior, i.e., of the constitutive matrices. For each element in a FE model, the direction of stiffest material direction is taken as reference direction with stiffest direction defined as the direction of largest longitudinal components in an optimal constitutive matrix, here termed $\left(\alpha_{1111}\right)_{\theta}$ with $\theta$ being the angle counter-clockwise from the common $x$-direction to a direction termed the $\theta$-direction.

The traditional lamina formulas are well suited for localizing $\theta$ for a specific element. With $\theta,\left(\alpha_{1111}\right)_{\theta}$ determined for all elements the available further physical information is calculated, applying practical parameters $\left(\alpha_{2}, \alpha_{3}, \alpha_{6}, \alpha_{7}\right)_{\theta}$ as evaluated for element $e$ in the specific reference direction $\theta_{e}$. In the present paper the non-dimensional, normalized practical quantities are given notation $\alpha$, as alternative to the often preferred notation $Q$ for corresponding dimensional quantities.

Although written in relation to $2 \mathrm{D}$ constitutive matrices, the approach is also valid for 2D structural stiffness matrices $[S], 2 \mathrm{D}$ structural flexibility matrices $[F]$, and 2D strength matrices in stress space $[H]$ or in strain space $[G]$. Also laminate stiffness sub-matrices 
and laminate flexibility sub-matrices may be visualized similarly.

The main readers in mind are researchers with interest in laminate formulation, but it is found necessary to give an introduction in Sections 2 and 3 to optimal constitutive matrices, before the application of laminate formulation is detailed in Section 4. Especially the discussion on "invariant" parameters should be noted. Finally, fields for constitutive matrices are exemplified with a suggested visualization for an optimal constitutive design obtained in [1].

\section{Separation of a non-dimensional constitutive ma- trix}

\section{from the local amount of material}

The distribution of material in a continuum is separated in two: firstly how much material to be used in a reference volume $V_{e}$ ? and secondly how this local material should be used to obtain an optimal local constitutive matrix ? By this separation a clear measure of the total amount of material/volume is possible.

The total amount of material volume $V$ is constrained and this constraint is assumed to be active, i.e., all material is assumed to be used. This assumption is essential for the obtained optimality criteria. With $\rho_{e}$ as local, non-dimensional design parameters for 
density, this constraint is written

$$
\sum_{e} \rho_{e} V_{e}=V
$$

with size limits

$0<\rho_{\min } \leq \rho_{e} \leq \rho_{\max } \leq 1$

and the major constraint is written

$$
g=\sum_{e} \rho_{e} V_{e}-V=0 \Rightarrow \partial g / \partial \rho_{e}=V_{e}
$$

The theory and procedures for iterative optimization to obtain the densities $\rho_{e}$ are shortly presented in Section 3.

The separated local (element $e$ ) constitutive matrix $\left[C_{e}\right]$ is

$$
\left[C_{e}\right]=\rho_{e} E_{0}\left[\widetilde{C}_{e}\right]=\rho_{e} E_{0}\left[\begin{array}{ccc}
\left(\widetilde{C}_{1111}\right)_{e} & \left(\widetilde{C}_{1122}\right)_{e} & \sqrt{2}\left(\widetilde{C}_{1112}\right)_{e} \\
\left(\widetilde{C}_{1122}\right)_{e} & \left(\widetilde{C}_{2222}\right)_{e} & \sqrt{2}\left(\widetilde{C}_{2212}\right)_{e} \\
\sqrt{2}\left(\widetilde{C}_{1112}\right)_{e} & \sqrt{2}\left(\widetilde{C}_{2212}\right)_{e} & 2\left(\widetilde{C}_{1212}\right)_{e}
\end{array}\right]
$$

where $E_{0}$ is a fixed value of modulus, $\rho_{e}$ a current local, non-dimensional density and $\left[\widetilde{C}_{e}\right]$ is a non-dimensional matrix, normalized to unit trace as well as to unit Frobenius norm. The discussion of this matrix is of primary interest.

\subsection{Constraint for the non-dimensional constitutive components}

The non-dimensional constitutive matrices are constrained to be symmetric and positive semi-definite and furthermore normalized such that the Frobenius norm $F_{e}=F\left(\left[\widetilde{C}_{e}\right]\right)$ is equal to 1 for all elements, here stated in terms of the squared norm $F_{e}^{2}$

$$
h_{e}=F_{e}^{2}-1=0
$$

With a design objective $\Phi$ and only the constraint (3), the necessary condition for optimality of the matrix components for $\left[\widetilde{C}_{e}\right]$ is proportionality between the gradients of 
the objective and the gradients of the constraint

$$
\frac{\partial \Phi}{\partial\left(\widetilde{C}_{i j k l}\right)_{e}}=\lambda \frac{\partial h_{e}}{\partial\left(\widetilde{C}_{i j k l}\right)_{e}}
$$

where for $2 \mathrm{D}$ problems $\left(\widetilde{C}_{i j k l}\right)_{e}$ is one of the six independent components of the constitutive matrix and $\lambda$ is a common factor for all six of these components, related to a specific constitutive matrix.

With $F_{e}^{2}$ defined as the sum of the squared components of the matrix $\left[\widetilde{C}_{e}\right]$ in $(2)$

$$
F_{e}^{2}=\left(\widetilde{C}_{1111}^{2}\right)_{e}+\left(\widetilde{C}_{2222}^{2}\right)_{e}+4\left(\widetilde{C}_{1212}^{2}\right)_{e}+2\left(\widetilde{C}_{1122}^{2}\right)_{e}+4\left(\widetilde{C}_{1112}^{2}\right)_{e}+4\left(\widetilde{C}_{2212}^{2}\right)_{e}
$$

and the gradients of the constraint $h_{e}=F_{e}^{2}-1=0$ are

$$
\begin{aligned}
& \frac{\partial h_{e}}{\partial\left(\widetilde{C}_{1111}\right)_{e}}=2\left(\widetilde{C}_{1111}\right)_{e}, \quad \frac{\partial h_{e}}{\partial\left(\widetilde{C}_{2222}\right)_{e}}=2\left(\widetilde{C}_{2222}\right)_{e} \\
& \frac{\partial h_{e}}{\partial\left(\widetilde{C}_{1212}\right)_{e}}=8\left(\widetilde{C}_{1212}\right)_{e}, \quad \frac{\partial h_{e}}{\partial\left(\widetilde{C}_{1122}\right)_{e}}=4\left(\widetilde{C}_{1122}\right)_{e}, \\
& \frac{\partial h_{e}}{\partial\left(\widetilde{C}_{1112}\right)_{e}}=8\left(\widetilde{C}_{1112}\right)_{e}, \quad \frac{\partial h_{e}}{\partial\left(\widetilde{C}_{2212}\right)_{e}}=8\left(\widetilde{C}_{2212}\right)_{e}
\end{aligned}
$$

The gradients of the objective, i.e. the left hand side of (4) for specific optimization objectives are derived subsequently.

\subsection{Compliance or total elastic energy as objective}

Compliance is, for design independent loads, equal to the total elastic energy $U$ (twice the total strain energy) and a gradient of $U$, say with respect to the constitutive components $\left(\widetilde{C}_{i j k l}\right)_{e}$, can be determined in a fixed strain field (fixed displacements field), see [2]

$$
\frac{\partial U}{\partial\left(\widetilde{C}_{i j k l}\right)_{e}}=-\left(\frac{\partial U}{\partial\left(\widetilde{C}_{i j k l}\right)_{e}}\right)_{f i x e d ~ s t r a i n}=-V_{e} \rho_{e} E_{0}\left(\frac{\partial \widetilde{u}_{e}}{\partial\left(\widetilde{C}_{i j k l}\right)_{e}}\right)_{f i x e d ~ s t r a i n}
$$

where $V_{e}$ is the volume in which we have constant strains $\{\epsilon\}_{e}$ and the constant constitutive matrix $[\widetilde{C}]_{e}$. Expanding the non-dimensional matrix product $\widetilde{u}_{e}=\{\epsilon\}_{e}^{T}[\widetilde{C}]_{e}\{\epsilon\}_{e}$ with 
strain vector $\{\epsilon\}_{e}^{T}=\left\{\epsilon_{11} \epsilon_{22} \sqrt{2} \epsilon_{12}\right\}_{e}$ gives

$$
\begin{aligned}
\widetilde{u}= & \left(\widetilde{C}_{1111}\right)_{e}\left(\epsilon_{11}^{2}\right)_{e}+\left(\widetilde{C}_{2222}\right)_{e}\left(\epsilon_{22}^{2}\right)_{e}+4\left(\widetilde{C}_{1212}\right)_{e}\left(\epsilon_{12}^{2}\right)_{e}+ \\
& 2\left(\widetilde{C}_{1122}\right)_{e}\left(\epsilon_{11}\right)_{e}\left(\epsilon_{22}\right)_{e}+4\left(\widetilde{C}_{1112}\right)_{e}\left(\epsilon_{11}\right)_{e}\left(\epsilon_{12}\right)_{e}+4\left(\widetilde{C}_{2212}\right)_{e}\left(\epsilon_{22}\right)_{e}\left(\epsilon_{12}\right)_{e}
\end{aligned}
$$

and the gradients are

$$
\begin{array}{ll}
\frac{\partial U}{\partial\left(\widetilde{C}_{1111}\right)_{e}}=\rho_{e} V_{e} E_{0}\left(\epsilon_{11}\right)_{e}\left(\epsilon_{11}\right)_{e}, & \frac{\partial U}{\partial\left(\widetilde{C}_{2222}\right)_{e}}=\rho_{e} V_{e} E_{0}\left(\epsilon_{22}\right)_{e}\left(\epsilon_{22}\right)_{e}, \\
\frac{\partial U}{\partial\left(\widetilde{C}_{1212}\right)_{e}}=4 \rho_{e} V_{e} E_{0}\left(\epsilon_{12}\right)_{e}\left(\epsilon_{12}\right)_{e}, & \frac{\partial U}{\partial\left(\widetilde{C}_{1122}\right)_{e}}=2 \rho_{e} V_{e} E_{0}\left(\epsilon_{11}\right)_{e}\left(\epsilon_{22}\right)_{e}, \\
\frac{\partial U}{\partial\left(\widetilde{C}_{1112}\right)_{e}}=4 \rho_{e} V_{e} E_{0}\left(\epsilon_{11}\right)_{e}\left(\epsilon_{12}\right)_{e}, & \frac{\partial U}{\partial\left(\widetilde{C}_{2212}\right)_{e}}=4 \rho_{e} V_{e} E_{0}\left(\epsilon_{22}\right)_{e}\left(\epsilon_{12}\right)_{e}
\end{array}
$$

\subsection{Multiple load cases and resulting optimality criterion for compliance optimizations}

With multiple load cases, all design independent, numbered $n=1,2, \ldots$ the gradients $(9)$ hold for each load case. The corresponding strains $\left(\epsilon_{11}\right)_{n},\left(\epsilon_{22}\right)_{n}$ and $\left(\epsilon_{12}\right)_{n}$ are all determined in the same coordinate system. Therefore, the simple optimization of minimizing a linear combination of compliances, expressed in the energies for each load case $U_{n}$, is

$$
\text { Minimizing } \quad U=\sum_{n} \eta_{n} U_{n} \quad \text { for } \quad h_{e}=F_{e}^{2}-1=0
$$

for given weight factors $\eta_{n}$, say with $\sum_{n} \eta_{n}=1$.

The design for the multiple load case that satisfies the optimality criterion is

$$
\left(\widetilde{C}_{i j k l}\right)_{e}=\lambda \sum_{n} \eta_{n}\left(\left(\epsilon_{i j}\right)_{e}\left(\epsilon_{k l}\right)_{e}\right)_{n}
$$

a simple optimal design result with $\lambda$ as a normalization factor. The case of a single load case is further simplified

$$
\left(\widetilde{C}_{i j k l}\right)_{e}=\lambda\left(\epsilon_{i j}\right)_{e}\left(\epsilon_{k l}\right)_{e}
$$


as seen directly by inserting (6) and (9) in (4). The extension from (12) to (11) follows directly with (9) interpreted for the specific load cases. For further detail see [2].

\subsection{Gradients and resulting optimality criterion}

\section{for single eigenfrequency optimization}

The local gradient of the Rayleigh quotient with respect to the components of the local constitutive matrix is simple when the mass distribution is unchanged (kinetic energies $T$ and $T_{e}$ unchanged), here with hat notation as an alternative to extended index of fixed displacements or fixed strains, see [3]

$$
\begin{aligned}
& \frac{\partial \omega^{2}}{\partial\left(\widetilde{C}_{i j k l}\right)_{e}}=\frac{\partial(U / T)}{\partial\left(\widetilde{C}_{i j k l}\right)_{e}}=\frac{\widehat{\partial(U / T})}{\partial\left(\widetilde{C}_{i j k l}\right)_{e}}=\frac{\left.\partial \widehat{\left(U_{e} / T\right.}\right)}{\partial\left(\widetilde{C}_{i j k l}\right)_{e}}=\frac{1}{T_{e}} \frac{{\widehat{\partial U_{e}}}_{\partial\left(\widetilde{C}_{i j k l}\right)_{e}}}{\rho_{e} V_{e} E_{0}} \frac{\widehat{\partial \widetilde{u}}_{e}}{\partial\left(\widetilde{C}_{i j k l}\right)_{e}} \\
& \text { with fixed strains in } \widetilde{u}_{e}=\{\epsilon\}_{e}^{T}[\widetilde{C}]_{e}\{\epsilon\}_{e}
\end{aligned}
$$

From the final relation in (13) then follows

$$
\begin{array}{ll}
\frac{\partial \omega^{2}}{\partial\left(\widetilde{C}_{1111}\right)_{e}}=\frac{\rho_{e} V_{e} E_{0}}{T_{e}}\left(\epsilon_{11}\right)_{e}\left(\epsilon_{11}\right)_{e}, & \frac{\partial \omega^{2}}{\partial\left(\widetilde{C}_{2222}\right)_{e}}=\frac{\rho_{e} V_{e} E_{0}}{T_{e}}\left(\epsilon_{22}\right)_{e}\left(\epsilon_{22}\right)_{e}, \\
\frac{\partial \omega^{2}}{\partial\left(\widetilde{C}_{1212}\right)_{e}}=4 \frac{\rho_{e} V_{e} E_{0}}{T_{e}}\left(\epsilon_{12}\right)_{e}\left(\epsilon_{12}\right)_{e}, & \frac{\partial \omega^{2}}{\partial\left(\widetilde{C}_{1122}\right)_{e}}=2 \frac{\rho_{e} V_{e} E_{0}}{T_{e}}\left(\epsilon_{11}\right)_{e}\left(\epsilon_{22}\right)_{e} \\
\frac{\partial \omega^{2}}{\partial\left(\widetilde{C}_{1112}\right)_{e}}=4 \frac{\rho_{e} V_{e} E_{0}}{T_{e}}\left(\epsilon_{11}\right)_{e}\left(\epsilon_{12}\right)_{e}, & \frac{\partial \omega^{2}}{\partial\left(\widetilde{C}_{2212}\right)_{e}}=4 \frac{\rho_{e} V_{e} E_{0}}{T_{e}}\left(\epsilon_{22}\right)_{e}\left(\epsilon_{12}\right)_{e}
\end{array}
$$

that except for a factor is identical to (9).

From (11), (12) and (14) it is seen that the optimality criterion for the discussed different 2D plane problems is for all of them similar to

$$
\left(\widetilde{C}_{i j k l}\right)_{e}=\left(\epsilon_{i j} \epsilon_{k l} /\left(\epsilon_{11}^{2}+\epsilon_{22}^{2}+2 \epsilon_{12}^{2}\right)\right)_{e}
$$

now written with the appropriate normalization, but without the weight factors $\eta_{n}$ in (11). 


\subsection{Multiple load cases fully stressed}

\section{for strength optimizations}

For strength design with free material subjected to multiple load cases, see details in [4]. A practical approach that is closely related to "fully stressed iterations" is chosen. The iteratively updated non-dimensional constitutive matrix is chosen as

$$
\left[\widetilde{C}_{e}\right]=\sum_{n}\left(\left(\eta_{e}\right)_{n}\left[\widetilde{C}_{e}\right]_{n}\right) \quad \text { with } \quad\left(\eta_{e}\right)_{n}=\frac{\left(u_{e}\right)_{n}}{\sum_{n}\left(u_{e}\right)_{n}} \Rightarrow \sum_{n}\left(\eta_{e}\right)_{n}=1
$$

where the weight factors for element $e$ are chosen by the relative elastic energy density $\left(u_{e}\right)_{n}$ in load case $n$, and each $\left[\widetilde{C}_{e}\right]_{n}$ are derived by optimality criteria and expressed in principal strains, see [5]. The heuristic procedure rapidly converges towards a design with an optimized strength that is measured by the maximum elastic energy density over the specified load cases and over the full continuum space.

\subsection{Proof of unit norms}

The result (15) shows that $\left[\widetilde{C}_{e}\right]$ is described by a dyadic product $\left[\widetilde{C}_{e}\right]=\{\alpha\}\{\alpha\}^{T}$. Then by definitions of trace and Frobenius norms follows, that the values of trace and Frobenius norms are always equal and $\left[\widetilde{C}_{e}\right]$ is semi-positive definite.

$$
\begin{aligned}
& \operatorname{trace}\left[\widetilde{C}_{e}\right]=\text { Frobenius }\left[\widetilde{C}_{e}\right]=\{\alpha\}^{T}\{\alpha\} \\
& \text { where }\{\alpha\}^{T}\{\alpha\}>0 \text { for }\{\alpha\} \neq\{0\}
\end{aligned}
$$

Omitting the index $e$ for element we proceed the discussion of the obtained constitutive matrix as described directly by the corresponding strain state(s). Although a constitutive matrix is not necessary obtainable as a dyadic product, this will be the case for the optimal constitutive matrix, where the important result in 2D plane problems with normalization 
to unit norms is

$$
[\widetilde{C}]=\{\alpha\}\{\alpha\}^{T} \text { with }\{\alpha\}^{T}=\left\{\begin{array}{lll}
\epsilon_{11} & \epsilon_{22} & \sqrt{2} \epsilon_{12}
\end{array}\right\} / \sqrt{\epsilon_{11}^{2}+\epsilon_{22}^{2}+2 \epsilon_{12}^{2}}
$$

That the optimal constitutive matrix of unit norms in 2D is described by only three parameters (the strain components) limits the possibilities for a matrix with normally up to 6 independent parameters.

Numerically the rate of change of the constitutive matrices are in each redesign of an optimization process limited by a non-dimensional step parameter $0 \leq \beta \leq 1$ similar to the design approach for strength optimization in [4] where $\beta=0.5$ and $\beta=0.1$ were used, i.e.,

$$
[\widetilde{C}]_{\text {new }}=\beta[\widetilde{C}]_{\text {from (18) }}+(1-\beta)[\widetilde{C}]_{\text {old }}
$$

The design approach is initiated with $[\widetilde{C}]_{0}=[I] / 3$, i.e., zero Poisson's ratio isotropic material, positive definite, non-dimensional and normalized. It is concluded that for a given strain state the optimized non-dimensional constitutive matrix is known with unit trace and Frobenius norm. Note, that with initial positive definite $[\widetilde{C}]$ it will for $\beta<1$ stay positive definite through the redesign iterations. Numerical value $\beta=0.2$ is applied for the visualized example in Section 5 , and even with this rather low $\beta$ value fast convergence is obtained.

\section{Optimality criteria for density variables}

\section{by design gradients}

For a design problem with the objective $\Phi$ to be minimized or maximized, subject to only one constraint $g=0$ for the optimized density design variables $\rho_{e}$ (index $e$ referring to 
element $e$ ), the necessary optimization criterion now involves the constraint $g=0$ in (1).

$$
\gamma_{e}=\frac{\partial \Phi}{\partial \rho_{e}} / \frac{\partial g}{\partial \rho_{e}}=\frac{\partial \Phi}{\partial \rho_{e}} / \frac{1}{V_{e}}=\gamma
$$

where the factor $\gamma$ is the same for all $\rho_{e}$ that are not at prescribed size limits.

The problems treated in the present paper are

- Minimum compliance $C=$ Minimum elastic energy $U$.

- Minimum linear combination of elastic energy for multiple load cases $U=\sum_{n} \eta_{n} U_{n}$.

- Maximum of first eigenfrequency (squared) $=\omega^{2}$.

- Maximum strength = Minimum of the maximum elastic energy density in a continuum applied to a number of load cases $n=1,2, \ldots$

For single load compliance design the optimality criterion is uniform elastic energy density, throughout the model, i.e. $\gamma_{e}=u_{e}=u$. Note, that for this most simple case the strength, as measured by the maximum elastic energy density, is simultaneously optimized.

The result for single load case extends directly for multiple load cases with linear combination compliance design with weight factors $\eta_{n}$ for each load case specified, i.e., $\gamma_{e}=\sum_{n} \eta_{n}\left(u_{e}\right)_{n}=\hat{u}_{e}=\hat{u}$, see $[2]$.

For multiple loads, strength design are assumed being obtained by uniform $\left(u_{\max }\right)_{e}$, i.e., by a fully stressed design, except at prescribed size limits. For isotropic material this is closely related to uniform von Mises stresses, see [4]. For anisotropic materials the elastic energy density is taken as a simple strength measure.

For eigenvalue related design as in free vibrations, the optimality criterion is uniform values of a weighted squared local frequency, i.e., $\gamma_{e}=\frac{1}{\rho_{e}} \frac{T_{e}}{T}\left(\omega_{e}^{2}-\omega^{2}\right)=\gamma$, see [3].

With these shortly stated results, the goal of the paper is to 
- Present practical iteration procedure that in $\simeq 10$ iterations $(\simeq 10$ finite element (FE) analyses) can determine designs of constant optimality criteria for all elements not at a size limit.

- Present a method for visualization of optimized design and response, with focus on the designed distributions of constitutive matrices.

- Described the mathematical tool for this visualization, based on classical laminate analysis.

\subsection{Numerical design procedure for density variables}

Assumed that a given design is specified by $\rho_{e}$ and analyzed to obtain $\Phi$ and $\frac{\partial \Phi}{\partial \rho_{e}}$. The individual ratios for $\gamma_{e}=\frac{\partial \Phi}{\partial \rho_{e}} \frac{1}{V_{e}}$ are different, and the numerical procedure for redesign should converge towards more uniform values of $\gamma_{e}$.

In cases with only positive values $\gamma_{e}>0$ a redesign is based on the weighted mean value $\bar{\gamma}>0$

$$
\bar{\gamma}=\sum_{e} \rho_{e} V_{e} \gamma_{e} / \sum_{e} \rho_{e} V_{e}
$$

and the redesign is from experience chosen to

$$
\left(\rho_{e}\right)_{\text {new }}=\left(\rho_{e}\right)_{\text {old }}\left(\frac{\gamma_{e}}{\bar{\gamma}}\right)^{q} F
$$

with $q=0.8$ and $F$ in an inner iteration determined such that the total volume constraint is satisfied, see [2] for details on the inner iterations.

In cases with negative as well as positive ratios $0>\left(\gamma_{e}\right)_{\min } \leq \gamma_{e} \leq\left(\gamma_{e}\right)_{\max }>0$, which is actual for the eigenfrequency optimization, the following heuristic procedure has been 
applied

For positive gradients $\left(\omega_{e}^{2}-\omega^{2}>0\right)$

$\left(\rho_{e}\right)_{\text {new }}=\left(\rho_{e}\right)_{\text {current }}\left(1+4.0 \gamma_{e} / \gamma_{\max }\right)^{0.8} \mathrm{~F}$

For negative gradients $\left(\omega_{e}^{2}-\omega^{2}<0\right)$

$\left(\rho_{e}\right)_{\text {new }}=\left(\rho_{e}\right)_{\text {current }}\left(1-0.8 \gamma_{e} / \gamma_{\min }\right)^{0.8} \mathrm{~F}$

where the values of $\gamma_{\min }<0, \gamma_{\max }>0$ are determined during the evaluation of the gradients. The specific values in (23) 4.0, 0.8, 0.8 are chosen from experience, acting as a kind of move-limits and influence the number of recursive redesigns (number of eigenvalue analysis). Again with $F$ in an inner iteration determined such that the total volume constraint is satisfied.

In general for both procedures (22) and (23) the size limits of the non-dimensional density variables

$$
0<\rho_{\min } \leq \rho_{e} \leq \rho_{\max } \leq 1
$$

are satisfied iteratively in an "inner" iteration loop without further analysis and sensitivity analysis. The factor $F$ thereby satisfy both the size limits (24) and the specified total amount of material/volume $V$ by $\sum_{e} \rho_{e} V_{e}=V$.

\section{Visualization of field of constitutive matrices}

Visualization of fields of $3 \times 3$, symmetric, positive definite constitutive matrices of unit norms are based on formulations from laminate theory. Practical parameters that often are termed invariants are valuable, but there seems to be a need for discussion of the property "invariant". 


\subsection{Use of laminate formula}

For anisotropic material the anisotropy should be visualized, but without going into all details of the six $2 \mathrm{D}$ components. A $2 \mathrm{D}$ material non-dimensional constitutive matrix $[\widetilde{C}]$ is given in a global $\mathrm{x}, \mathrm{y}$ coordinate system with the $\mathrm{x}$-direction as the reference direction by

$$
[\widetilde{C}]=\left[\begin{array}{ccc}
\alpha_{1111} & \alpha_{1122} & \sqrt{2} \alpha_{1112} \\
\alpha_{1122} & \alpha_{2222} & \sqrt{2} \alpha_{2212} \\
\sqrt{2} \alpha_{1112} & \sqrt{2} \alpha_{2212} & 2 \alpha_{1212}
\end{array}\right]_{x}
$$

with the assumed condition that $[\widetilde{C}]$ is positive definite and the trace of the positive diagonal elements is normalized to unity, i.e.,

$$
\alpha_{1111}+\alpha_{2222}+2 \alpha_{1212}=1
$$

These conditions then hold in any rotated coordinate system. A physical description of the constitutive matrix is of major interest, so the direction of largest longitudinal material stiffness must be located.

According to laminate theory $\alpha_{1111}$ as a function of rotation, termed $\left(\alpha_{1111}\right)_{\theta}$, is given by the six components in the $x$ reference coordinate system, here chosen in a form linear in trigonometric factors,

$$
\begin{aligned}
\left(\alpha_{1111}\right)_{\theta}= & \left(\alpha_{1111}+\alpha_{2222}\right)_{x} / 2+\left(\alpha_{2}\right)_{x} \cos (2 \theta)-\left(\alpha_{3}\right)_{x}(1-\cos (4 \theta))+ \\
& \left(\alpha_{6}\right)_{x} 2 \sin (2 \theta)+\left(\alpha_{7}\right)_{x} \sin (4 \theta)
\end{aligned}
$$


where the practical parameters are defined by

$$
\begin{aligned}
& \left(\alpha_{2}\right)_{x}=\left(\alpha_{1111}-\alpha_{2222}\right)_{x} / 2 \\
& \left(\alpha_{3}\right)_{x}=\left(\alpha_{1111}+\alpha_{2222}-2\left(\alpha_{1122}+2\left(\alpha_{1212}\right)\right)_{x} / 8\right. \\
& \left(\alpha_{6}\right)_{x}=\left(\alpha_{1112}+\alpha_{2212}\right)_{x} / 2 \\
& \left(\alpha_{7}\right)_{x}=\left(\alpha_{1112}-\alpha_{2212}\right)_{x} / 2
\end{aligned}
$$

For orthotropic materials $\alpha_{6}=\alpha_{7}=0$ in specific directions, but for the free material this will not always be the case, so we analyze the more general case. Several extrema for $\left(\alpha_{1111}\right)_{\theta}$ may exist in the interval $0 \leq \theta<\pi$. To locate the maximum of $\left(\alpha_{1111}\right)_{\theta}$, the function (27) is numerically evaluated at a number of $\theta$ values (here chosen with increments $\Delta \theta=\pi / 1800)$. This can be done for each elements and $\theta_{e}$ is then the angle for the largest value $\left(\alpha_{1111}\right)_{\theta}$. The values of $\left(\alpha_{1111}\right)_{\theta}$ has an upper bound of 1 and a lower bound of $1 / 3$. This follows from the trace being 1 , and having positive eigenvalues in this interval. This then also follows for the non-dimensional longitudinal stiffness. For high values of $\left(\alpha_{1111}\right)_{\theta}$ a single fiber direction is approached and for lower values of $\left(\alpha_{1111}\right)_{\theta}$ an isotropic material with zero Poisson's ratio material is approached.

Similar to (27) the remaining constitutive components with the $\theta$-direction as reference direction may be evaluated by

$$
\begin{aligned}
\left(\alpha_{2222}\right)_{\theta}= & \left(\alpha_{1111}+\alpha_{2222}\right)_{x} / 2-\left(\alpha_{2}\right)_{x} \cos (2 \theta)-\left(\alpha_{3}\right)_{x}(1-\cos (4 \theta))- \\
& \left(\alpha_{6}\right)_{x} 2 \sin (2 \theta)+\left(\alpha_{7}\right)_{x} \sin (4 \theta) \\
\left(\alpha_{1122}\right)_{\theta}= & \left(\alpha_{1122}\right)_{x}+\left(\alpha_{3}\right)_{x}(1-\cos (4 \theta))-\left(\alpha_{7}\right)_{x} \sin (4 \theta) \\
\left(\alpha_{1212}\right)_{\theta}= & \left(\alpha_{1212}\right)_{x}+\left(\alpha_{3}\right)_{x}(1-\cos (4 \theta))-\left(\alpha_{7}\right)_{x} \sin (4 \theta) \\
\left(\alpha_{1112}\right)_{\theta}= & \left.\left(\alpha_{2}\right)_{x} \sin (2 \theta) x / 2-\left(\alpha_{3}\right)_{x} \sin (4 \theta)\right)+\left(\alpha_{6}\right)_{x} \cos (2 \theta)+\left(\alpha_{7}\right)_{x} \cos (4 \theta) \\
\left(\alpha_{2212}\right)_{\theta}= & \left.\left(\alpha_{2}\right)_{x} \sin (2 \theta) x / 2+\left(\alpha_{3}\right)_{x} \sin (4 \theta)\right)+\left(\alpha_{6}\right)_{x} \cos (2 \theta)-\left(\alpha_{7}\right)_{x} \cos (4 \theta)
\end{aligned}
$$


all this well known from laminate theory.

\subsection{Discussion on "invariant" parameters}

The definitions of $\left.\left(\alpha_{2}\right)_{\theta},\left(\alpha_{3}\right)_{\theta},\left(\alpha_{6}\right)_{\theta}\right),\left(\alpha_{7}\right)_{\theta}$ with reference to a specific $\theta$-direction is defined by

$$
\begin{aligned}
& \left(\alpha_{2}\right)_{\theta}=\left(\alpha_{1111}-\alpha_{2222}\right)_{\theta} / 2 \\
& \left(\alpha_{3}\right)_{\theta}=\left(\alpha_{1111}+\alpha_{2222}-2\left(\alpha_{1122}+2\left(\alpha_{1212}\right)\right)_{\theta} / 8\right. \\
& \left(\alpha_{6}\right)_{\theta}=\left(\alpha_{1112}+\alpha_{2212}\right)_{\theta} / 2 \\
& \left(\alpha_{7}\right)_{\theta}=\left(\alpha_{1112}-\alpha_{2212}\right)_{\theta} / 2
\end{aligned}
$$

and their numerical values may be different from the parameters in (28). The following relations are derived by inserting (27) and (29) in (30)

$$
\begin{array}{ll}
\left(\alpha_{2}\right)_{\theta}=\left(\alpha_{2}\right)_{x} \cos (2 \theta)+\left(\alpha_{6}\right)_{x} 2 \sin (2 \theta) & \left(\left(\alpha_{2}\right)_{\theta}=\left(\alpha_{2}\right)_{x} \text { for } \theta=0 \text { and } \pi\right) \\
\left(\alpha_{3}\right)_{\theta}=\left(\alpha_{3}\right)_{x} \cos (4 \theta)+\left(\alpha_{7}\right)_{x} \sin (4 \theta) & \left(\left(\alpha_{3}\right)_{\theta}=\left(\alpha_{3}\right)_{x} \text { for } \theta=0 \text { and } \pi\right) \\
\left(\alpha_{6}\right)_{\theta}=\left(\alpha_{6}\right)_{x} \cos (2 \theta)-\left(\alpha_{2}\right)_{x} \sin (2 \theta) / 2 & \left(\left(\alpha_{6}\right)_{\theta}=\left(\alpha_{6}\right)_{x} \text { for } \theta=0 \text { and } \pi\right) \\
\left(\alpha_{7}\right)_{\theta}=\left(\alpha_{7}\right)_{x} \cos (4 \theta)-\left(\alpha_{3}\right)_{x} \sin (4 \theta) & \left(\left(\alpha_{7}\right)_{\theta}=\left(\alpha_{7}\right)_{x} \text { for } \theta=0 \text { and } \pi\right)
\end{array}
$$

Therefore the practical parameters $\alpha_{2}, \alpha_{3}, \alpha_{6}, \alpha_{7}$ are not only material parameters but also depend on the reference axis chosen. Note especially the sign change $\left(\alpha_{3}\right)_{\theta}=-\left(\alpha_{3}\right)_{x}$ for $\theta=\frac{\pi}{4}$ and $\frac{3 \pi}{4}$, implying that for each element, reference axes always exit with both positive and negative $\alpha_{3}$ (being zero for the isotropic case).

Material orthotropy imply zero of the following parameter combinations, expressed 
both with reference to $\mathrm{x}$ and $\theta$ as main axis

$$
\begin{aligned}
& z_{x}=\left(\alpha_{7}\right)_{x}\left(\alpha_{2}\right)_{x}^{2}-4\left(\alpha_{7}\right)_{x}\left(\alpha_{6}\right)_{x}^{2}-4\left(\alpha_{6}\right)_{x}\left(\alpha_{3}\right)_{x}\left(\alpha_{2}\right)_{x} \\
& z_{\theta}=\left(\alpha_{7}\right)_{\theta}\left(\alpha_{2}\right)_{\theta}^{2}-4\left(\alpha_{7}\right)_{\theta}\left(\alpha_{6}\right)_{\theta}^{2}-4\left(\alpha_{6}\right)_{\theta}\left(\alpha_{3}\right)_{\theta}\left(\alpha_{2}\right)_{\theta}
\end{aligned}
$$

If $z_{x}$ is zero, then the material is orthotropic and $z_{\theta}$ is then also zero, because the condition (32) holds in any coordinate system. The derived functions (31) fulfills this, by setting $\left(\alpha_{6}\right)_{x}=\left(\alpha_{7}\right)_{x}=0$.

The conclusion from the present analysis is that the parameters (30) as well as (28) should be termed practical parameters instead of invariant parameters.

\subsection{Important anisotropy quantities}

It is suggested for the constitutive matrices of an optimized design to present the following five distributions

- Largest longitudinal stiffness by $\left(\alpha_{1111}\right)_{\theta e}$ for all elements $e$ in a color plot, noting the limits $1 / 3 \leq\left(\alpha_{1111}\right)_{\theta e} \leq 1$ with $1 / 3$ for isotropy with zero Poisson's ratio and with 1 for unidirectional fiber.

- Direction of largest longitudinal stiffness by directional lines, noting the limits $0 \leq$ $\theta_{e} \leq \pi$. May be combined with the color plot above.

- Level of anisotropy by $2\left(\alpha_{2}\right)_{\theta e}=\left(\alpha_{1111}\right)_{\theta e}-\left(\alpha_{2222}\right)_{\theta e}$ for all elements $e$ in a color plot, noting the limits 0 and 1 with 1 for high level of anisotropy and 0 for symmetry.

- Relative importance of shear stiffness by $8\left(\alpha_{3}\right)_{\theta e}$ for all elements $e$ in a color plot. High shear stiffness corresponds to negative values of $8\left(\alpha_{3}\right)_{\theta e}$, i.e. $4 \alpha_{1212}>\alpha_{1111}+$ $\alpha_{2222}-2 \alpha_{1122}$, as seen in (30). Alternatively, $\alpha_{1212}$ may be directly visualized. 
- Test for material orthotropy by $z_{e}$ for all elements $e$ in a color plot. Only places with $z_{e}=0$ have orthotropic material. The color plot relates to a scaled, squared test quantity with a lower limit to identity orthotropy.

\subsection{Other matrices with similar rotational transformations}

The visualizations of the present paper written in relation to $2 \mathrm{D}$ constitutive matrices, are also valid for $2 \mathrm{D}$ structural stiffness matrices $[S]$, 2D structural flexibility matrices $[F]$, and $2 \mathrm{D}$ strength matrices in stress space $[H]$ or in strain space $[G]$. Also laminate stiffness sub-matrices and laminate flexibility sub-matrices may be visualized similarly.

\section{Visualization of example from optimal anisotropy}

In [1] a cantilever (with fixed material at the tip) is optimized to maximize the first eigenfrequency. Without specifying here the details of analysis and optimization by iterative redesign, we visualize in Figure 1 the obtained constitutive matrices, as suggested above in Section 4.3.

Some clarifying comments to Figure 1 must be given. At the tip a strip of full material density is prescribed in order to have a meaningful model. Close to this strip the optimal density reach the minimum density, this area being the same for all four figures is shown in white. This might be a little misleading relative to Figure 1d, because here white is used to indicate material classified as orthotropic (no material/low density is also orthotropic). The black direction lines in Figure 1a are intented to be illustrative. The model has 16384 elements with individual directions and limitations to a reasonable number of continuous lines are necessary. The lines in Figure 1a are therefore only for illustration and in [1] for two examples, they are shown more clearly on a white background. 
a) Largest longitudinal stiffness by $\left(\alpha_{1111}\right)_{\theta}$

b) Level of anisotropy by $2\left(\alpha_{2}\right)_{\theta}$
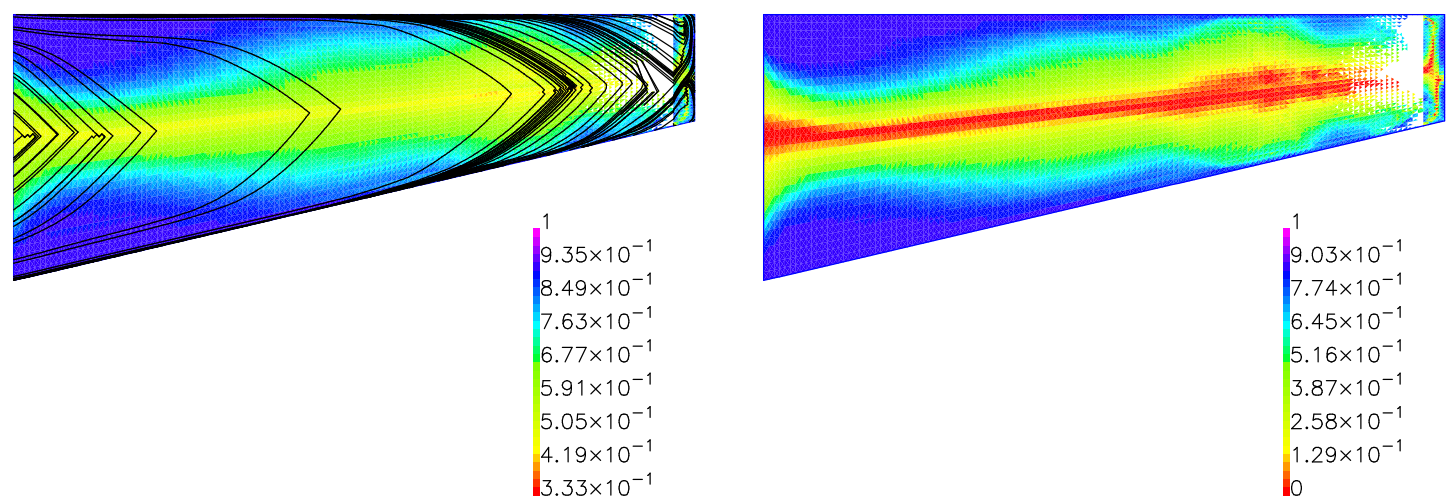

c) Relative shear stiffness by $8\left(\alpha_{3}\right)_{\theta}$

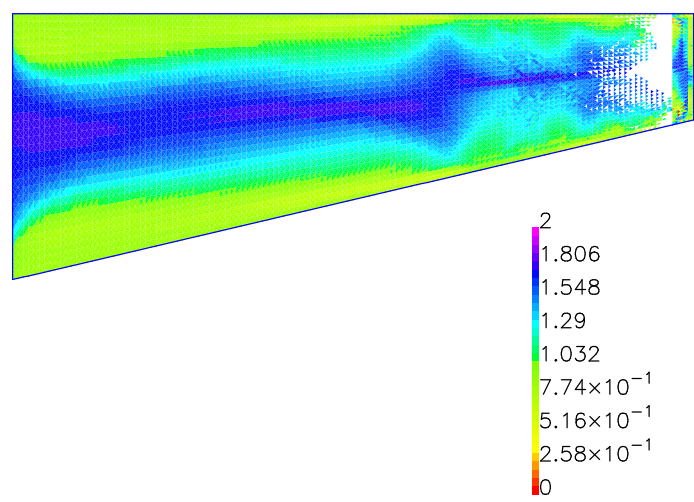

d) Orthotropic material, only if $z_{\theta}^{2}=0$

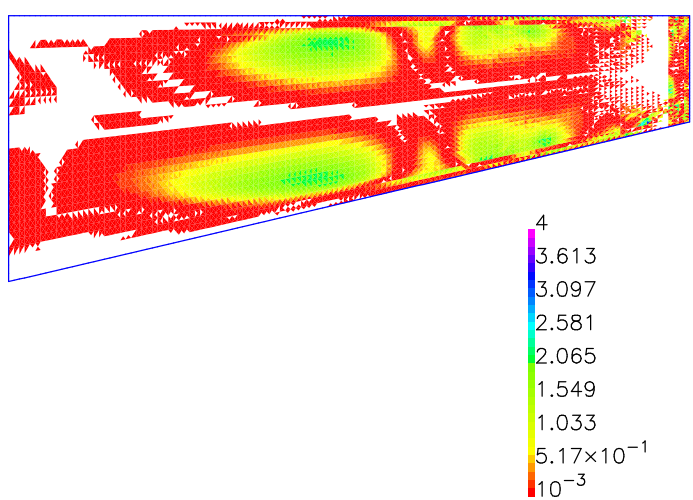

Figure 1: Visualization of distributions for constitutive matrices added direction of largest longitudinal stiffness in Figure 1a. The white spots near the tip of fixed material are places of minimum material density, being the same in all Figures 1a-d. The further white areas in Figure 1d contains materials classified as orthotropic. 
Note, that the quantities in Figures 1a-c are measured in individual rotated coordinate systems which reference axes are visualized by the direction lines in Figure 1a.

For largest longitudinal stiffness in Figure 1a we see blue color (close to the value 0.85) at the upper and lower boundaries, yellow color (close to 0.45) at the "beam axis" and green color between these zones. All this as expected in relation to the simplest bending eigenmode. For direction of largest longitudinal stiffness added in Figure 1a, the 45 degrees at the "beam axis" and parallel to the upper and lower boundaries also agree with simple bending of a cantilever.

The distribution of level of anisotropy is visualized by $2\left(\alpha_{2}\right)_{\theta}$ in Figure $1 \mathrm{~b}$ and show small relative values of $\left(\alpha_{2222}\right)_{\theta}$ by blue color close to upper and lower boundaries, i.e.,

high level of anisotropy. Close to symmetry $\left(\alpha_{2222}\right)_{\theta} \simeq\left(\alpha_{1111}\right)_{\theta}$ by red color close to the "beam axis".

The relative importance of shear stiffness is visualized by distribution of $8\left(\alpha_{3}\right)_{\theta}$ in Figure 1c. For high shear stiffness, relative to a chosen reference main axis, this quantity will be negative. For the present case only positive values are found with the reference axes corresponding to $\theta_{e}$, i.e., with the directions of largest longitudinal stiffness.

The distribution of possible orthotropy is visualized by a scaled $z_{\theta}^{2}$ in Figure 1d, where the zero limit is set to 0.001. The white areas (away from the tip) are thus areas of material orthotropy, without showing the directions of orthotropy.

\section{Conclusion}

Visualization of results from optimal design may not be too complicated in traditional size, shape or topology design, but in free material optimization (FMO) constitutive matrices in the continuum or structural space are part of the obtained design. A visualization 
of such distribution of matrices for $2 \mathrm{D}$ problems with 6 different matrix components is demonstrated.

From laminate analysis, the formulation for rotational transformation is applied and is found useful. Practical parameters that usual are stated as invariants are an important part of this formulation, but the notion invariants needs to be discussed, because it only relates to a specific reference direction. The visualized distributions of physical quantities are stiffest material direction, material stiffest longitudinal constitutive component, level of anisotropy, absolute or relative shear stiffness and orthotropy test.

Optimal design of material distribution is often effectively obtained by design iterations based on a stated optimality criterion. It is recently found that the optimal constitutive matrices (the anisotropy) are simply related to the actual strain field(s). Since this is not well known, it is chosen to shortly describe the theory behind this result as an introductory to the visualization aspects. Although four different optimization problems are involved, the theoretical results and the numerical applied procedures are rather similar. In all cases the constitutive components are directly given by strain states that in redesigns are iteratively determined. The constitutive matrix may for optimized solutions be written as a dyadic product. The density distributions follow for the three static problems by optimality criteria of uniform elastic energy density that may be weighted in relation to multiple load cases. For strength optimization this can be interpreted as fully stressed designs.

\section{References}

[1] P. Pedersen and N. L. Pedersen. Distributed material density and anisotropy for optimized eigenfrequency of 2D continua. Struct. Multidisc. Optim., 51(5):1067-1076, 
2015.

[2] P. Pedersen and N. L. Pedersen. Optimality criteria for the components of anisotropic constitutive matrix. Struct. Multidisc. Optim., 50:181-191, 2014.

[3] P. Pedersen and N. L. Pedersen. A note on eigenfrequency sensitivities and structural eigenfrequency optimization based on local sub-domain frequencies. Struct. Multidisc. Optim., 49(4):559-568, 2014.

[4] P. Pedersen and N. L. Pedersen. On strength design using free material subjected to multiple load cases. Struct. Multidisc. Optim., 47(1):7-17, 2013.

[5] M. P. Bendsøe, J. M. Guedes, R. B. Haber, P. Pedersen, and J. E. Taylor. An analytical model to predict optimal material properties in the context of optimal structural design. J. Applied Mechanics, 61:930-937, 1994. 